\title{
Applications of Pressure Perturbation Calorimetry to Study Factors Contributing to the Volume Changes upon Protein Unfolding
}

\author{
Pranav P. Pandharipande ${ }^{1,3}$ and George I. Makhatadze $e^{1,2, *}$ \\ ${ }^{1}$ Center for Biotechnology and Interdisciplinary Studies, ${ }^{2}$ Department of Biological Sciences and \\ ${ }^{3}$ Howard P. Isermann Department of Chemical and Biological Engineering, Rensselaer \\ Polytechnic Institute, Troy, NY 12180
}

* Corresponding Author: George I. Makhatadze, Center for Biotechnology and Interdisciplinary Studies, Rensselaer Polytechnic Institute, $1108^{\text {th }}$ Street, Troy, NY 12180 USA Phone: (518)276-4417 E-mail:makhag@ rpi.edu

Note: The authors declare no competing financial interest. 


\begin{abstract}

\section{Background}

Pressure perturbation calorimetry (PPC) is a biophysical method that allows direct determination of the volume changes upon conformational transitions in macromolecules.
\end{abstract}

\title{
Scope of this Review
}

This review provides novel details of the use of PPC to analyze unfolding transitions in proteins. The emphasis is made on the data analysis as well as on the validation of different structural factors that define the volume changes upon unfolding. Four case studies are presented that show the application of these concepts to various protein systems.

\section{Major Conclusions}

The major conclusions are:

1. Knowledge of the thermodynamic parameters for heat induced unfolding facilitates the analysis of the PPC profiles.

2. The changes in the thermal expansion coefficient upon unfolding appear to be temperature dependent.

3. Substitutions on the protein surface have negligible effects on the volume changes upon protein unfolding.

4. Structural plasticity of proteins defines the position dependent effect of amino acid substitutions of the residues buried in the native state. 
5. Small proteins have positive volume changes upon unfolding which suggests difference in balance between the cavity/void volume in the native state and the hydration volume changes upon unfolding as compared to the large proteins that have negative volume changes.

\section{General Significance}

The information provided here gives a better understanding and deeper insight into the role played by various factors in defining the volume changes upon protein unfolding. 


\section{Introduction}

How a linear sequence of amino acids folds into an intricate and biologically active threedimensional structure of a protein has captivated the scientific community ever since the myoglobin structure was reported [1,2]. Multiple physico-chemical forces such as hydrogen bond, hydrophobic interaction, van der Waals interaction, disulfide bridges and electrostatic interactions have been widely discussed for their role in defining the protein stability (see e.g. [37]. Much of the studies have been exploring the effect of fundamental environmental parameter, temperature $(\mathrm{T})$ on protein stability (defined as the Gibbs energy difference between unfolded and native states $\left.\Delta G=G_{U^{-}} G_{N}\right)$. However, much less has been done to explore the dependence of the stability of proteins on the other equally fundamental environmental parameter, hydrostatic pressure $(\mathrm{P})$. This is important to understand the physico-chemical principles that define the stability of proteins in general and in particular for the organisms that live in the deep sea, the socalled barophiles $[8,9]$.

The pressure dependence of the protein stability is defined by the volume changes upon unfolding, $\Delta \mathrm{V}$ :

$$
\Delta V=V_{U}-V_{N}=\left(\frac{\partial \Delta G}{\partial P}\right)_{T}
$$

where $\mathrm{V}_{\mathrm{U}}$ and $\mathrm{V}_{\mathrm{N}}$ are the volumes of the unfolded and native states, respectively. If $\Delta \mathrm{V}$ is negative, increase in hydrostatic pressure will, according to Le Châtelier's principle, lead to a decrease in protein stability, while positive values of $\Delta \mathrm{V}$ will lead to an increase in stability. There are two major factors contributing to the $\Delta \mathrm{V}$ of protein unfolding. The first factor is the imperfection in the packing of the native proteins, i.e. well documented presence of cavities of 
voids [10-12]. The second factor is the volume changes of solvent water upon hydrating the protein groups exposed due to the unfolding [13]:

$$
\Delta V=V_{\text {voids }}+V_{\text {Hydration }}
$$

The exact magnitudes of these effects, especially the hydration of newly exposed accessible surface area due denaturation, are still under debate [14-16].

Until recently, the volume changes upon unfolding have been determined indirectly from the pressure dependence of equilibrium constant (see e.g. ref [16]). Introduction of the commercial instruments to perform pressure perturbation calorimetry (PPC) experiments [17] and subsequent development of data analysis formalism [18] provided the protein folding community the ability to directly determine $\Delta \mathrm{V}$ of conformational transitions. These measurements combined with other biophysical methods assessing thermodynamic (e.g. DSC) and structural (e.g. circular dichroism and fluorescence spectroscopies, NMR, SAXS) properties of proteins and supported by computational modeling allowed better understanding of the properties of proteins that contribute to the pressure dependence of protein stability [18-40].

In this paper we report several robust approaches to analyze the PPC profiles of proteins and report four novel case studies of protein systems that clarify the importance of various factors in defining the volume changes upon protein unfolding. The article is organized as follows. After the Materials and Methods section, we present a brief discussion of the PPC experiment and foundation for a two-state analysis. This is followed by case studies of four different protein systems for which PPC experiments were performed. Each case highlights 
certain aspects of data analysis and more importantly, probes various structural aspects of proteins that define their volume changes upon unfolding.

\section{Materials and Methods}

\subsection{TrpZip Peptide}

TRPZIP4 with sequence GEWTWDDATKTWTWTE-NH 2 was synthesized using standard Fmoc chemistry as described previously [41]. The peptide has a molecular weight of $2013 \mathrm{Da}$ and extinction coefficient $\left(\varepsilon_{280,0.1 \%}\right)$ of 11.41 optical units. The partial specific volume, $\bar{V}_{p r}$, was calculated to be $0.70 \mathrm{~cm}^{3} / \mathrm{g}$ based on the amino acid composition as described previously [42]. Both DSC and PPC experimental procedures were performed using TRPZIP4 concentrations between 0.5 and $3.5 \mathrm{mg} / \mathrm{ml}$. Peptide purification was done using a C18 reverse phase HPLC column (Discovery Bio Wide pore C18, $10 \mu \mathrm{m}$, Supelco Sigma-Aldrich, Bellefonte, PA) and a 0 to $100 \%$ acetonitrile gradient in the presence of $0.05 \%$ trifluoroacetic acid (TFA). Pooled fractions were lyophilized and then washed with Milli-Q (Millipore, Billerica, MA) water. This procedure was repeated at least three times to remove residual TFA which could potentially interfere with spectroscopic and calorimetric measurements. MALDI-TOF (Bruker Ultraflex III, Bruker Daltonics, Billerica, MA) was carried out to determine the molecular weight and purity of the samples. The peptide concentrations were determined using an UV-Vis spectrophotometer (Hitachi U-2900). Prior to the calorimetric experiments, all samples were

thoroughly dialyzed using Spectra/Por 1,000 Da molecular weight cutoff membrane (18x11.5 $\mathrm{mm}$ ) into $20 \mathrm{mM}$ sodium phosphate buffer $\mathrm{pH}$ 7.0. Three dialysis changes of 4 hours each were performed. 


\subsection{BPTI, ubiquitin and acylphosphatase variants}

Bovine pancreatic trypsin inhibitor (BPTI) was purchased from United States Biochemical Corp. (Cleveland, OH, USA, cat. No. 11388) and used without further purification.

The constructs for ubiquitin and protocol for expression and purification has been described by us previously $[43,44]$.

The constructs for ACP-wt and ACP-GA2 have been described previously [45]. QuickChange site directed mutagenesis Kit (Stratagene) was used to introduce mutations Ala at position corresponding to Ile13, Phe22, Leu35, Val39, Val47, Leu51 and Val58 in the overall background of ACP-GA2. The presence of the desired mutation in the gene of interest in the cloning vector, pGia, was confirmed by direct DNA sequencing (Eurofins MWG Operon). Expression plasmids were transformed into Escherichia coli BL21(DE3) competent cells (Novagen, Madison, WI) using the heat shock method. The cells were grown in 1 L LB medium containing $100 \mu \mathrm{g} / \mathrm{ml}$ ampicillin at $37^{\circ} \mathrm{C}$ to an optical density of 0.8 at $600 \mathrm{~nm}$ and induced by adding $1 \mathrm{mM}$ (final concentration) of IPTG. The induction of protein expression was carried out for 6 hours. Cells were harvested at $7500 \mathrm{x} \mathrm{g}$ and the pellet was suspended in $15 \mathrm{~mL}$ of Ni-NTA denaturing lysis buffer B (Novagen) (8M Urea, $0.1 \mathrm{M} \mathrm{NaH}_{2} \mathrm{PO}_{4}, 0.01 \mathrm{M}$ Tris- $\mathrm{HCl}, \mathrm{pH}$ 8) and stored at $-20^{\circ} \mathrm{C}$ until further use. The frozen pellet was thawed and passed through a French pressure cell and the lysed cells were centrifuged at $39,000 \mathrm{xg}$ for 45 minutes at $4{ }^{\circ} \mathrm{C}$ to remove cell debris. The supernatant was diluted with Ni-NTA denaturing lysis buffer B (Novagen) in 1:1 ratio and applied to Ni-NTA resin (Novagen) column twice. Stationary phase was washed with denaturing wash buffer pH 6.25 (8M Urea, $0.1 \mathrm{M} \mathrm{NaH}_{2} \mathrm{PO}_{4}, 0.01 \mathrm{M}$ Tris- $\left.\mathrm{HCl}\right)$. Additional wash with pH 5.9 wash buffer ( $8 \mathrm{M}$ Urea, $0.1 \mathrm{M} \mathrm{NaH}_{2} \mathrm{PO}_{4}, 0.01 \mathrm{M}$ Tris- $\mathrm{HCl}$ ) was done until 
optical density at $280 \mathrm{~nm}$ was less than 0.01 o.u. The protein was eluted using $\mathrm{pH} 4.45$ elution buffer (8M Urea, $0.1 \mathrm{M} \mathrm{NaH}_{2} \mathrm{PO}_{4}, 0.01 \mathrm{M}$ Tris- $\mathrm{HCl}$ ). The eluted protein was thoroughly dialyzed against 1L 5\% acetic acid and applied onto a G-50 Sephadex gel filtration column $(2.5 \times 150 \mathrm{~cm})$ equilibrated in $5 \%$ acetic acid. Protein containing fractions were pooled, lyophilized and stored at $-20^{\circ} \mathrm{C}$. Purity of the protein was evaluated by SDS-PAGE. In addition the molecular mass of the proteins were determined by MALDI-TOF mass spectrophotometer (Bruker Ultraflex III, Bruker Daltonics, Billerica, MA) and found to be within 1-4 Da of the theoretically expected.

All proteins were thoroughly dialyzed using 3,500 Da molecular weight cutoff dialysis tubing prior to calorimetric experiments. All discussed PPC and DSC experiments were performed in $20 \mathrm{mM}$ glycine buffer in $\mathrm{pH}$ range from 1.75 to 3.55 .

\subsection{Differential scanning calorimetry (DSC)}

DSC experiments were performed from 5 to $115^{\circ} \mathrm{C}$ at a heating rate $90 \mathrm{deg}$ per hour on a VP-DSC (GE-Microcal) following a protocol discussed elsewhere.[46] The acquired raw data was converted to heat capacity profiles using Origin lab software (Origin, Northampton, MA). The heat capacity profiles were analyzed according to a two-state model as described previously (ref [46] using in-house written script for nonlinear regression software, NLREG.

\subsection{Circular dichroism spectroscopy $(C D)$}

CD measurements were performed using a JASCO J-710 spectropolarimeter and a $1 \mathrm{~mm}$ rectangular quartz cuvette. The temperature was controlled at $20^{\circ} \mathrm{C}$ using a Peltier effect cell holder. Prior to the protein scans (200 to $260 \mathrm{~nm}$ ), scans of the buffer alone were performed to 
determine the baseline signal. The ellipticity $(\theta)$ was converted to mean residue ellipticity $\left([\theta]_{\mathrm{MR}}\right)$ using the following equation,

$[\theta]_{M R}=\frac{\theta \cdot M W r}{l \cdot c_{p r}}$

3.

where 1 is the optical length of the cell, $\mathrm{c}_{\mathrm{pr}}$ is the protein concentration and $\mathrm{MWr}$ is the average mass per amino acid residue for each variant. 


\section{Results and Discussion}

\subsection{Experimental Determination of Thermal Expansion Coefficient of Proteins and Volume}

\section{Changes upon Unfolding}

In a PPC experiment, the thermal expansion coefficient is determined from the heat effects $\Delta Q_{b u f / p r}(T)$, produced in the sample cell (containing protein in a buffer) relative to the reference cell (that has that same buffer alone) as both cells are subjected to rapid and small amount of perturbations in hydrostatic pressure $(\Delta \mathrm{P} \sim 5 \mathrm{~atm})$ under isothermal conditions [17]. The heat effects, $\Delta Q_{b u f / p r}(T)$, are proportional to the thermal expansion coefficient as:

$$
\alpha(T)=\alpha_{\mathrm{H}_{2} \mathrm{O}}(T)-\frac{\Delta Q_{\mathrm{H}_{2} \mathrm{O} / b u f}(T)}{T \cdot \Delta P \cdot v_{\text {cell }}}-\frac{\Delta Q_{\text {buf } / p r}(T)}{T \cdot \Delta P \cdot v_{\text {cell }} \cdot c_{p r} \cdot V_{p r}}
$$

where $v_{\text {cell }}$ is the volume of the calorimetric cell, $V_{p r}$ is the partial specific volume of protein and $\mathrm{c}_{\mathrm{pr}}$ is the protein concentration, $\alpha_{\mathrm{H}_{2} \mathrm{O}}(T)$ is the thermal expansion coefficient of pure water, and $\Delta Q_{\mathrm{H}_{2} \mathrm{O} / \text { buf }}(T)$ reflects to the difference in thermal expansion coefficients of water and buffer. The latter is measured for each individual buffer in a separate PPC experiments. Pressure perturbation is performed at different temperatures to obtain the temperature dependence of $\alpha(\mathrm{T})$.

The experimentally measured function of $\alpha(T)$ for protein unfolding can be analyzed to determine the volume changes upon unfolding, $\Delta V_{p r} / V_{p r}$. The analytical experssion for the analysis of PPC data according to a two-state model was derived by us previously [18, 40]: 
$\alpha(T)=F_{N}(T) \cdot \alpha_{N}(T)+F_{U}(T) \cdot \alpha_{U}(T)+\frac{K_{e q}(T)}{\left(1+K_{e q}(T)\right)^{2}} \frac{\Delta H(T)}{R T^{2}} \frac{\Delta V_{p r}}{V_{p r}}$

Where $F_{N}(T)$ is the fraction of protein molecules in the folded state, $F_{U}(T)$ is the fraction of protein molecules in the unfolded state, $K_{e q}(T)$ is the equilibrium constant, $\Delta H(T)$ is the enthalpy of unfolding, and $a_{N}(T)$ and $a_{U}(T)$ are the temperature dependences of the thermal expansion coefficients for the native and unfolded state, respectively.

In cases where the trasition is very broad it is possible to simultaneously analyse the DSC and PPC profiles by combining equation 5 and a two state equatuion for the heat capacity profile:

$C_{p}(T)=F_{N}(T) \cdot C_{p, N}(T)+F_{U}(T) \cdot C_{p, U}(T)+\frac{K_{e q}(T)}{\left(1+K_{e q}(T)\right)^{2}} \frac{\Delta H(T)^{2}}{R T^{2}}$

6.

where $\mathrm{C}_{\mathrm{p}, \mathrm{N}}(T)$ and $\mathrm{C}_{\mathrm{p}, \mathrm{U}}(T)$ are the temperature dependences of the heat capcities for the native and unfolded state, respectively (ref [46]). 


\subsection{Temperature Dependence of Thermal Expansion Coefficients of Proteins}

The temperature dependences of the thermal expansion coefficients for the native and unfolded state $a_{N}(T)$ and $a_{U}(T)$ are non-linear functions. It was found that the unfolded state baseline can be calculated from the amino acid composition using an additivity scheme:

$\alpha_{U, \text { calc }}(T)=\frac{\sum_{i} v_{i}(T) \cdot \alpha_{i}(T)}{\sum_{i} v_{i}(T)}$

7.

where $v_{i}(T)$ and $\alpha_{i}(T)$ are the partial molar volume and coefficient of thermal expansion of side chain $i$, respectively. Comparison shows an excellent agreement between the experimentally measured and calculated using equation 7 thermal expansion coefficient (ref [39]).

Similarly, the thermal expansion coefficient for the native state $a_{N}(T)$ also shows nonlinear dependence on temperature, although this dependence is not as steep as for the unfolded state, particularly in the temperature range between $0^{\circ} \mathrm{C}$ and $\sim 60^{\circ} \mathrm{C}$ (see ref [38] and case studies below).

\subsection{Case Study 1: Ubiquitin and variants}

Ubiquitin (Ubq) is a 76 amino acid protein of $\alpha / \beta$-fold (Figure 1A). We used several Ubq variants to address two main questions. 1. What are the effects of substitutions on the surface of the native protein on the volume changes upon protein unfolding? 2 . What are the effects of substitutions at the buried location in the native protein on the volume changes upon protein unfolding? 
To address the first question, we measured the volume changes upon unfolding of several variants of Ubq with different substitutions on the surface in the native state (see Figure 1B for sequence comparison). We find that in all cases the values of $\Delta \mathrm{V}_{\mathrm{pr}} / \mathrm{V}_{\mathrm{pr}}$ remain very similar suggesting that the residues on the surface of the native protein do not contribute much to the volume changes upon unfolding (Figure 1C). This result is not new and has been expected based on the current understanding of factors contributing to protein volume changes upon protein unfolding, because residues on the surface remain solvent exposed when the protein unfolds. However, this is probably the first direct and rather comprehensive experimental validation for the negligible contribution of surface exposed residues to the volume changes upon unfolding (see also the results for ACP below).

To address the second question, we made two substitutions at the buried positions in the native state. In both cases, fully buried larger Val was substituted to smaller Ala. These substitutions are expected to create larger cavity/void in the native state of the Ubq. The resulting Ubq variants $\mathrm{V} 5 \mathrm{~A}$ and V17A show more negative volume changes upon unfolding (Figure 1C) consistent with the expectations that cavity creating mutations should lead to a decrease in $\Delta \mathrm{V}_{\mathrm{pr}} / \mathrm{V}_{\mathrm{pr}}$. Interestingly, the magnitude of the volume changes and the temperature dependencies of $\Delta \mathrm{V}_{\mathrm{pr}} / \mathrm{V}_{\mathrm{pr}}$ for the two variants are the same suggesting the effects of Val-to-Ala substitutions at two different positions of Ubq are very similar. This does not have to be a general property as similar ACP variants do not show such effect (see below).

\subsection{Case Study 2: ACP and variants}


Acylphosphatase (ACP) is a globular protein of 98 residues (Figure 2A). We used this protein to test the generality of volume change due to the substitutions on the surface or interior of the native protein as observed for Ubq.

We first compared the volume changes of the wild type ACP and ACP variant (GA2) that is $\sim 10^{\circ} \mathrm{C}$ more stable [45]. This variant has optimized surface charge-charge interactions that is achieved by introducing 5 substitutions in the charged positions (see Figure 2B for sequences). Substitutions in charged residues will also have an effect on the overall electrostatic interactions of the protein with the solvent, so if there are any effects of electrostriction on the volume changes upon unfolding, the comparison of wt-ACP and GA2-ACP will provide a direct estimate of the magnitude of these effects. Figure $2 \mathrm{C}$ compares the volume changes upon unfolding for these two proteins. It is evident that substitutions in the charged residues on the surface of the native protein did not lead to any significant changes in $\Delta \mathrm{V}_{\mathrm{pr}} / \mathrm{V}_{\mathrm{pr}}$. There is a clear similarity with the effects of the surface substitutions on the $\Delta \mathrm{V}_{\mathrm{pr}} / \mathrm{V}_{\mathrm{pr}}$ values in ubiquitin (see Figure 1C). This allows us to conclude that the residues on the surface of the native state of a protein do not contribute to the volume changes upon unfolding.

We then examined the effects of substitutions at the buried positions on the volume changes in ACP. Seven different sites were chosen to replace large nonpolar residues to a smaller Ala. The substitutions included three Val-to-Ala substitutions (V39A, V47A and V58A), two Leu-to-Ala substitutions (L35A and L51A), one Ile-to-Ala substitution (I13A) and one Phe-to-Ala substitution (F22A). The sites for substitutions are scattered throughout the structure (see Figure 2A) and do not seem to introduce major structural perturbations (see Figure S1). 
These large nonpolar-to-Ala substitutions in the ACP lead, as expected [43, 44], to a decrease in protein stability. The decrease in stability leads to technical difficulties in defining the $\alpha_{N}(T)$ and $\alpha_{U}(T)$ functions required for the two-state analysis. Two major factors help overcome these issues. First is the ability to predict the temperature dependence of the $\alpha_{\mathrm{U}}(T)$ from the amino acid composition [39]. Figure 3 shows the PPC profiles for the L35A variant of ACP. The protein becomes unfolded at all temperatures at very low $\mathrm{pH}$ values. For example, at pH 2.25 the thermal expansion coefficient of L35A variant as measured by PPC does not show any transition suggesting that at this $\mathrm{pH}$ the protein is unfolded. Importantly, the experimentally measured $\alpha(\mathrm{T})$ for L35A at $\mathrm{pH} 2.25$ overlaps with the $\alpha_{\mathrm{U}}(\mathrm{T})$ calculated based on previously developed approach [39]. The second important factor is that the amino acid substitutions have little effect on the $\alpha_{N}(T)$. In an extensive study of over nine different proteins and several of their variants, we have shown that $\alpha_{N}(T)$ appears to be defined by the topology of a given protein fold [38]. Thus one can use the estimated $\alpha_{\mathrm{N}}(\mathrm{T})$ of a more stable variant to fit the data of a less stable one. An example of this is shown in Figure 3, where it clearly shows that the $\alpha_{N}(T)$ for the ACP-GA2 (a more stable protein) can be used to define the $\alpha_{N}(T)$ for the L35A variant. Combining these two estimates for $\alpha_{\mathrm{N}}(\mathrm{T})$ and $\alpha_{\mathrm{U}}(\mathrm{T})$ functions allows a very good fit of the data to a two state model and subsequently a rather accurate estimate of the $\Delta \mathrm{V}_{\mathrm{pr}} / \mathrm{V}_{\mathrm{pr}}$ values upon protein unfolding (see Figure 2C).

In Ubq the two Val-to-Ala substitutions had very similar effect on the $\Delta \mathrm{V}_{\mathrm{pr}} / \mathrm{V}_{\mathrm{pr}}$ values. However, in ACP the effects of Val-to-Ala are very different (Figure 2C). V39A has the largest decrease in volume while V58A has very small effect and V47A has volume change between V39A and V58A. Other substitutions that are designed to replace even larger side chains with Ala also produce rather mixed effect (Figure 2C). The substitution that was expected to produce 
the largest effect, F22A, does not show any significant changes in the $\Delta \mathrm{V}_{\mathrm{pr}} / \mathrm{V}_{\mathrm{pr}}$ values relative the reference protein ACP-GA2. The L35A variant has the $\Delta \mathrm{V}_{\mathrm{pr}} / \mathrm{V}_{\mathrm{pr}}$ values comparable to V39A and very different from ACP-GA2, but the I13A and L51A variants have much smaller difference from the $\Delta \mathrm{V}_{\mathrm{pr}} / \mathrm{V}_{\mathrm{pr}}$ values for ACP-GA2. So the question then is why Val-to-Ala substitutions in Ubq produced uniform effects on the $\Delta \mathrm{V}_{\mathrm{pr}} / \mathrm{V}_{\mathrm{pr}}$ values (Figure 1C) while substitutions on ACP did not (Figure 2C). One possible explanation might be the structural plasticity of these two proteins. Ubq, being an extremely conserved structural protein, is very rigid and any structural perturbation in its core does not allow for much of structural reorganization. Hence, similar substitution at different buried positions produces similar effects on the $\Delta \mathrm{V}_{\mathrm{pr}} / \mathrm{V}_{\mathrm{pr}}$ values. $\mathrm{ACP}$ is an enzyme and its sequence is not very conserved suggesting a larger structural plasticity of this protein. As a result, substitutions in the core of this protein lead to relaxation and rearrangement in the structure to better accommodate these amino acid substitutions. This in turn will lead to a different effect on the $\Delta \mathrm{V}_{\mathrm{pr}} / \mathrm{V}_{\mathrm{pr}}$ values from similar substitutions at different buried positions.

\subsection{Case Study 3: BPTI}

BPTI is a 57 amino acid residue and contains 3 disulfide bridges that are critical for protein stability (Figure 4A). Reduction of disulfide bonds leads to complete unfolding of the protein. This fact was used by us previously to measure the temperature dependence of the expansivity of the unfolded state of this protein [39]. It was found that $\alpha_{U}(T)$ can be well described by a third order polynomial. Furthermore, it was shown that the temperature dependence of the thermal expansion coefficient for the unfolded state of a protein can be predicted from the amino acid composition and thermal expansion coefficients of individual 
amino acid residues (see equation 7 and ref [39]. BPTI is a very thermostable protein [47], and this allowed the estimation of the temperature dependence of $\alpha_{N}(T)$ for the native state. It was shown that $\alpha_{\mathrm{N}}(\mathrm{T})$ decreases with the increase of temperature up to $\sim 60^{\circ} \mathrm{C}$ and then levels off [38] with the functional dependence well described by an exponential function of temperature as discussed in detail previously [38]. Knowing the functional dependences of $\alpha_{N}(T)$ and $\alpha_{U}(T)$ allows us to fit the PPC profiles for BPTI to a two state model. Figure 4B shows the results of the global fit of six independent PPC experiments.

There are two interesting observations. The first observation is that the volume changes upon unfolding of BPTI are positive. Our recent structural calculations [12] of the fraction of void volume in the native proteins showed that smaller proteins are better packed than larger proteins. This finding suggested that small proteins might have less negative or even positive values of $\Delta \mathrm{V}_{\mathrm{pr}} / \mathrm{V}_{\mathrm{pr}}$ in qualitative agreement with positive volume changes observed for BPTI. The second observation is that there is a decrease in magnitude of positive $\Delta \mathrm{V}_{\mathrm{pr}} / \mathrm{V}_{\mathrm{pr}}$ with increase in temperature. One possible explanation of this effect is that $\Delta \alpha=\alpha_{U}-\alpha_{N}$ is not a constant function of temperature as was previously assumed. Indeed, the $\alpha_{N}(T)$ and $\alpha_{U}(T)$ functions for BPTI and other proteins (see also Figures 1C and 2C) are not parallel and are expected to cross at higher temperatures. So it is conceivable that the decrease in $\Delta \mathrm{V}_{\mathrm{pr}} / \mathrm{V}_{\mathrm{pr}}$ with temperature in BPTI is a direct manifestation of this effect.

\subsection{Case Study 4: TrpZip}

The tryptophan zipper (TrpZip) is a 16-residue peptide of sequence GEWTWDDATKTWTWTE-NH 2 that forms a beta-hairpin (Figure 5A). The structure has been solved by NMR and subsequent biophysical studies found TrpZip to be highly soluble and 
monomeric in aqueous solutions, has relatively high thermostability of $75^{\circ} \mathrm{C}$, the folding/unfolding reaction is reversible and thermodynamics of unfolding can be well described by a two-state model [41]. Figure 5B shows the experimentally measured, using PPC, dependence of the thermal expansion coefficient of TrpZip on temperature. The enthalpy of unfolding of this small peptide is relatively small [41] and results in a rather broad transition that can be well seen from the DSC profile (Figure 5B). Furthermore, the unfolding transition is not complete even at the highest experimentally achievable temperature. Because of these the fit of the thermal expansion coefficient according to a two-state model (equation 5) requires additional constrains such as knowledge of enthalpy and heat capacity changes upon unfolding and the temperature dependence of $\alpha_{U}(T)$. This was done by: 1. simultaneously fitting the DSC (equation 6) and PPC (equation 5) profiles and 2. by using the calculated, from the amino acid composition (see equation 7), $\alpha_{U}(\mathrm{~T})$ function. The resulting fit suggests that $\Delta \mathrm{V}_{\mathrm{pr}} / \mathrm{V}_{\mathrm{pr}}$ for TrpZip is small and positive. The positive value for the volume changes upon unfolding for small proteins has been suggested by us earlier [12] and the results for BPTI and TrpZip provide direct experimental support for this prediction.

\section{Concluding Remarks}

The four case studies discussed above provide novel details concerning the use of PPC for the analysis of conformational transitions in proteins and also shed more light on factors defining the volumetric properties of proteins.

1. Knowledge of the thermodynamic parameters for heat induced unfolding facilitates the analysis of the PPC profiles. This is particularly important for the transitions that are broad (i.e. low enthalpy) and also for the transitions that have small volume changes. 
2. The temperature dependence of the $\alpha_{N}(T)$ and $\alpha_{U}(T)$ are relatively well defined and can be used as restrains in the analysis of PPC profiles. The case studies presented here reinforce our previous findings and provide direct use of explicitly defined functional relationships to analyze PPC profiles

3. The changes in thermal expansion coefficient upon unfolding appear to be temperature dependent. Both the difference in temperature dependencies of $\alpha_{N}(T)$ and $\alpha_{U}(T)$ and temperature dependence of volume changes upon unfolding of BPTI suggest that $\Delta \alpha$ decreases with the increase in temperature

4. Substitutions on the protein surface have negligible effects on the volume changes upon protein unfolding. This appears to be a general property as it was validated with multiple variants of two different proteins, Ubq and ACP.

5. Structural plasticity of proteins defines the position dependent effect of amino acid substitutions of the residues buried in the native state. Proteins that have a more evolutionary conserved sequence (e.g. ubiquitin) appear to have less structural plasticity to adjust to the substitutions. Conversely, proteins that are not well conserved in sequence (e.g. acylphosphatase) are more likely to readjust the structure in response to cavity-creating substitutions.

6. Small proteins have positive volume changes upon unfolding which suggests a difference in balance between the cavity/void volume in the native state and hydration volume changes upon unfolding, as compared to the large proteins that have negative volume changes. The positive volume changes were previously suggested from the analysis of packing densities of proteins [12] and two example provided here provide initial support for this prediction. 
The future direction in the study of pressure stability of proteins will include quantitative predictions of volume changes upon unfolding using explicit computational modeling of conformational states, detailed understanding of the role of hydration in defining protein volume changes, and ultimately computational de-novo design of proteins with given volumetric properties. 


\section{Acknowledgements}

This work was supported by the grants from the US National Science Foundation (CHE-1145407 and CHE-1506468). 


\section{References}

[1] J.C. Kendrew, G. Bodo, H.M. Dintzis, R. Parrish, H. Wyckoff, D.C. Phillips, A threedimensional model of the myoglobin molecule obtained by x-ray analysis, Nature, 181 (1958) 662-666.

[2] K.A. Dill, J.L. MacCallum, The protein-folding problem, 50 years on, Science, 338 (2012) 1042-1046.

[3] K.A. Dill, Dominant forces in protein folding, Biochemistry, 29 (1990) 7133-7155.

[4] W. Kauzmann, Some factors in the interpretation of protein denaturation, Advances in protein chemistry, 14 (1959) 1-63.

[5] C.N. Pace, B.A. Shirley, M. McNutt, K. Gajiwala, Forces contributing to the conformational stability of proteins, Faseb J, 10 (1996) 75-83.

[6] R.L. Baldwin, Temperature dependence of the hydrophobic interaction in protein folding, Proceedings of the National Academy of Sciences, 83 (1986) 8069-8072.

[7] G.I. Makhatadze, P.L. Privalov, Energetics of protein structure, Adv Protein Chem, 47 (1995) $307-425$.

[8] K. Horikoshi, Barophiles: deep-sea microorganisms adapted to an extreme environment, Current opinion in microbiology, 1 (1998) 291-295.

[9] F.M. Lauro, D.H. Bartlett, Prokaryotic lifestyles in deep sea habitats, Extremophiles, 12 (2008) 15-25.

[10] F.M. Richards, Areas, volumes, packing and protein structure, Annual review of biophysics and bioengineering, 6 (1977) 151-176.

[11] J. Liang, K.A. Dill, Are proteins well-packed?, Biophys J, 81 (2001) 751-766. 
[12] C.R. Chen, G.I. Makhatadze, ProteinVolume: calculating molecular van der Waals and void volumes in proteins, Bmc Bioinformatics, 16 (2015).

[13] K. Gekko, Volume and Compressibility of Proteins, Subcell Biochem, 72 (2015) 75-108.

[14] T.V. Chalikian, R.B. Macgregor, Origins of pressure-induced protein transitions, Journal of molecular biology, 394 (2009) 834-842.

[15] W. Kauzmann, Thermodynamics of unfolding, Nature, 325 (1987) 763-764.

[16] C.A. Royer, Revisiting volume changes in pressure-induced protein unfolding, Biochimica et Biophysica Acta (BBA)-Protein Structure and Molecular Enzymology, 1595 (2002) 201209.

[17] L.-N. Lin, J.F. Brandts, J.M. Brandts, V. Plotnikov, Determination of the volumetric properties of proteins and other solutes using pressure perturbation calorimetry, Analytical biochemistry, 302 (2002) 144-160.

[18] K.L. Schweiker, G.I. Makhatadze, Use of pressure perturbation calorimetry to characterize the volumetric properties of proteins, Methods in enzymology, 466 (2009) 527-547.

[19] H. Seemann, R. Winter, C.A. Royer, Volume, expansivity and isothermal compressibility changes associated with temperature and pressure unfolding of Staphylococcal nuclease, J Mol Biol, 307 (2001) 1091-1102.

[20] H. Herberhold, C.A. Royer, R. Winter, Effects of chaotropic and kosmotropic cosolvents on the pressure-induced unfolding and denaturation of proteins: an FT-IR study on staphylococcal nuclease, Biochemistry, 43 (2004) 3336-3345.

[21] R. Ravindra, R. Winter, Pressure Perturbation Calorimetry: A New Technique Provides Surprising Results on the Effects of Co-solvents on Protein Solvation and Unfolding Behaviour, ChemPhysChem, 5 (2004) 566-571. 
[22] P.D. Heerklotz, Pressure perturbation calorimetry, Methods Mol Biol, 400 (2007) 197-206.

[23] S. Lee, H. Heerklotz, T.V. Chalikian, Effects of buffer ionization in protein transition volumes, Biophys Chem, 148 (2010) 144-147.

[24] J.D. Batchelor, A. Olteanu, A. Tripathy, G.J. Pielak, Impact of protein denaturants and stabilizers on water structure, Journal of the American Chemical Society, 126 (2004) 19581961.

[25] D.G. Barrett, C.M. Minder, M.U. Mian, S.J. Whittington, W.J. Cooper, K.M. Fuchs, A. Tripathy, M.L. Waters, T.P. Creamer, G.J. Pielak, Pressure perturbation calorimetry of helical peptides, Proteins, 63 (2006) 322-326.

[26] S. Benjwal, O. Gursky, Pressure perturbation calorimetry of apolipoproteins in solution and in model lipoproteins, Proteins, 78 (2010) 1175-1185.

[27] S. Jayaraman, R. Jasuja, M.N. Zakharov, O. Gursky, Pressure perturbation calorimetry of lipoproteins reveals an endothermic transition without detectable volume changes. Implications for adsorption of apolipoprotein to a phospholipid surface, Biochemistry, 50 (2011) 3919-3927.

[28] S. Suladze, M. Kahse, N. Erwin, D. Tomazic, R. Winter, Probing volumetric properties of biomolecular systems by pressure perturbation calorimetry (PPC)--the effects of hydration, cosolvents and crowding, Methods, 76 (2015) 67-77.

[29] M. Erlkamp, S. Grobelny, R. Winter, Crowding effects on the temperature and pressure dependent structure, stability and folding kinetics of Staphylococcal Nuclease, Phys Chem Chem Phys, 16 (2014) 5965-5976.

[30] Y. Zhai, L. Okoro, A. Cooper, R. Winter, Applications of pressure perturbation calorimetry in biophysical studies, Biophys Chem, 156 (2011) 13-23. 
[31] P.L. Chong, R. Ravindra, M. Khurana, V. English, R. Winter, Pressure perturbation and differential scanning calorimetric studies of bipolar tetraether liposomes derived from the thermoacidophilic archaeon Sulfolobus acidocaldarius, Biophys J, 89 (2005) 1841-1849.

[32] G. Panick, R. Malessa, R. Winter, G. Rapp, K.J. Frye, C.A. Royer, Structural characterization of the pressure-denatured state and unfolding/refolding kinetics of staphylococcal nuclease by synchrotron small-angle X-ray scattering and Fourier-transform infrared spectroscopy, J Mol Biol, 275 (1998) 389-402.

[33] G. Desai, G. Panick, M. Zein, R. Winter, C.A. Royer, Pressure-jump studies of the folding/unfolding of trp repressor, J Mol Biol, 288 (1999) 461-475.

[34] C. Krywka, C. Sternemann, M. Paulus, M. Tolan, C. Royer, R. Winter, Effect of osmolytes on pressure-induced unfolding of proteins: a high-pressure SAXS study, Chemphyschem, 9 (2008) 2809-2815.

[35] J.B. Rouget, M.A. Schroer, C. Jeworrek, M. Puhse, J.L. Saldana, Y. Bessin, M. Tolan, D. Barrick, R. Winter, C.A. Royer, Unique features of the folding landscape of a repeat protein revealed by pressure perturbation, Biophys J, 98 (2010) 2712-2721.

[36] R. Kitahara, K. Hata, A. Maeno, K. Akasaka, M.S. Chimenti, E.B. Garcia-Moreno, M.A. Schroer, C. Jeworrek, M. Tolan, R. Winter, J. Roche, C. Roumestand, K. Montet de Guillen, C.A. Royer, Structural plasticity of staphylococcal nuclease probed by perturbation with pressure and pH, Proteins, 79 (2011) 1293-1305.

[37] P.P. Pandharipande, G.I. Makhatadze, Thermal expansivities of peptides, polypeptides and proteins as measured by pressure perturbation calorimetry, Methods, 76 (2015) 61-66. 
[38] D. Vasilchuk, P.P. Pandharipande, S. Suladze, J.M. Sanchez-Ruiz, G.I. Makhatadze, Molecular determinants of expansivity of native globular proteins: a pressure perturbation calorimetry study, J Phys Chem B, 118 (2014) 6117-6122.

[39] A.D. Tsamaloukas, N.K. Pyzocha, G.I. Makhatadze, Pressure perturbation calorimetry of unfolded proteins, J Phys Chem B, 114 (2010) 16166-16170.

[40] K.L. Schweiker, V.W. Fitz, G.I. Makhatadze, Universal convergence of the specific volume changes of globular proteins upon unfolding, Biochemistry, 48 (2009) 10846-10851.

[41] W.W. Streicher, G.I. Makhatadze, Calorimetric evidence for a two-state unfolding of the beta-hairpin peptide trpzip4, Journal of the American Chemical Society, 128 (2006) 30-31.

[42] G.I. Makhatadze, S.J. Gill, P.L. Privalov, Partial molar heat capacities of the side chains of some amino acid residues in aqueous solution. The influence of the neighboring charges, Biophys Chem, 38 (1990) 33-37.

[43] V.V. Loladze, D.N. Ermolenko, G.I. Makhatadze, Heat capacity changes upon burial of polar and nonpolar groups in proteins, Protein Sci, 10 (2001) 1343-1352.

[44] V.V. Loladze, D.N. Ermolenko, G.I. Makhatadze, Thermodynamic consequences of burial of polar and non-polar amino acid residues in the protein interior, J Mol Biol, 320 (2002) 343-357.

[45] A.V. Gribenko, M.M. Patel, J. Liu, S.A. McCallum, C. Wang, G.I. Makhatadze, Rational stabilization of enzymes by computational redesign of surface charge-charge interactions, Proc Natl Acad Sci U S A, 106 (2009) 2601-2606.

[46] M.M. Lopez, G.I. Makhatadze, Differential scanning calorimetry, Methods Mol Biol, 173 (2002) 113-119. 
[47] G.I. Makhatadze, K.S. Kim, C. Woodward, P.L. Privalov, Thermodynamics of BPTI folding, Protein Sci, 2 (1993) 2028-2036. 


\section{FIGURE LEGENDS}

Figure 1. Structure and thermodynamics of Ubq variants. Panel A: Cartoon structure of ubiquitin (PDB: 1UBQ) showing the substitution sites at buried positions as van der Waals spheres (yellow - V5A, green - V17A). Panel B: Sequence alignment of the ubiquitin variants. Highlighted are the sites of substitution on the surface of the native protein relative to the human ubiquitin. Panel C: Temperature dependence of the $\Delta \mathrm{V}_{\mathrm{pr}} / \mathrm{V}_{\mathrm{pr}}$ for the Ubq variants as measured by PPC. The variants with amino acid substitution on the surface show very similar values: bovine ubiquitin $(\bullet)$, wt* $(\boldsymbol{\Lambda})$, wt\# $(\square), 6 H-w t^{*}(\nabla)$, GA1 $(\diamond)$, GA3 $(\diamond)$. Single-site substitutions V5A $(\bullet)$ and V17A ( $\square$ ) were made in the background of wt\# and show significantly more negative volume changes upon unfolding.

Figure 2. Structure and thermodynamics of ACP variants. Panel A: Cartoon structure of human acylphosphatase (PDB: $2 \mathrm{~K} 7 \mathrm{~K}$ ) showing the substitution sites at buried positions as van der Waals spheres (colors matching the colors of symbols in Panel C). Panel B: Sequence alignment of ACP variants. Highlighted are the sites of substitution relative to the wt ACP, underlined are the single-site substitutions in the GA2 background. Panel C: Temperature dependence of the $\Delta \mathrm{V}_{\mathrm{pr}} / \mathrm{V}_{\mathrm{pr}}$ for ACP variants as measured by PPC. Open and filled circles correspond to the WT and GA2 variants respectively. Single-site substitutions at the buried positions were all made in the background of GA2 variant: I13A ( $\nabla)$, F22A ( $\square)$, L35A ( $\square)$, V39A $(\diamond)$, V47A $(\Delta)$, L51A $(\diamond)$, V58A $(\diamond)$. 
Figure 3. Analysis of the PPC experiments for L35A-variant of ACP. Experimental unfolding profiles show negative volume changes upon unfolding: pH 3.31 ( $\square)$, pH $3.37(\diamond)$, $\mathrm{pH} 3.49(\Delta)$ and $\mathrm{pH} 3.58(\square)$. The black line is the fit of experimental data to a twostate model (equation 5). The red and green lines correspond to the temperature dependencies of native and unfolded functions, respectively, as defined in equation 5. For comparison the PPC profile of ACP-GA2 at pH 3.00 (o) and ACP-L35A at $\mathrm{pH} 2.25(\nabla)$ are also shown. Note the agreement of the unfolded function with the profile at $\mathrm{pH} 2.25$ where the protein is unfolded. The agreement of the fitted native state function with the native state of ACP-GA2 is also remarkable. The $\Delta \mathrm{V}_{\mathrm{pr}} / \mathrm{V}_{\mathrm{pr}}$ values obtained from the fit are shown in Figure $2 \mathrm{C}$ as yellow squares.

Figure 4. Structure and thermodynamics of BPTI. Panel A: Cartoon structure of BPTI (PDB: 6PTI). Panel B: Analysis of the PPC experiments for BPTI. Experimental unfolding profiles show positive volume changes upon unfolding: $\mathrm{pH} 1.75$ (o), 2.00 $(\nabla), 2.5(\square), 2.75(\diamond), 3.00(\Delta)$ and $3.5(\square)$. The black line is the fit of experimental data to a two-state model (equation 5). The red and green lines correspond to the temperature dependencies of native and unfolded functions, respectively, as defined in equation 5 and using thermodynamic data from ref [47]. The $\Delta \mathrm{V}_{\mathrm{pr}} / \mathrm{V}_{\mathrm{pr}}$ values obtained from the fit were $+0.0059(\mathrm{pH} \mathrm{1.75}),+0.0056(\mathrm{pH} \mathrm{2.00)},+0.0057(\mathrm{pH} 2.5)$, $+0.0053(\mathrm{pH} 2.75),+0.0049(\mathrm{pH} 3.00)$ and $+0.0039(\mathrm{pH} 3.5)$ with the estimated error of $10 \%( \pm 0.0005)$. 
Figure 5. Structure and thermodynamics of TrpZip. Panel A: Cartoon structure of TrpZip (PDB: 1LE3). Panel B: Analysis of the PPC experiments for TrpZip. Experiments were performed in triplicate and average values $(\bullet)$ with corresponding standard deviations are shown. The black line is the fit of experimental data to a two-state model (equation 5) with the following parameters: $\mathrm{T}_{\mathrm{m}}=75^{\circ} \mathrm{C}, \Delta \mathrm{H}=90 \mathrm{~kJ} / \mathrm{mol}$, $\Delta \mathrm{V}_{\mathrm{pr}} / \mathrm{V}_{\mathrm{pr}}=+0.008 \pm 0.002, \Delta \mathrm{C}_{\mathrm{p}}=1.4 \mathrm{~kJ} /(\mathrm{mol} \mathrm{K})$ [41]. The red, green, yellow, and blues lines correspond to the temperature dependencies of native, unfolded, progress and excess functions of thermal expansion coefficient, respectively, as defined in equation 5. Thick black line shows the corresponding DSC profiles with the Y-axes (heat capacity) on the right. 


\section{Figure 1}

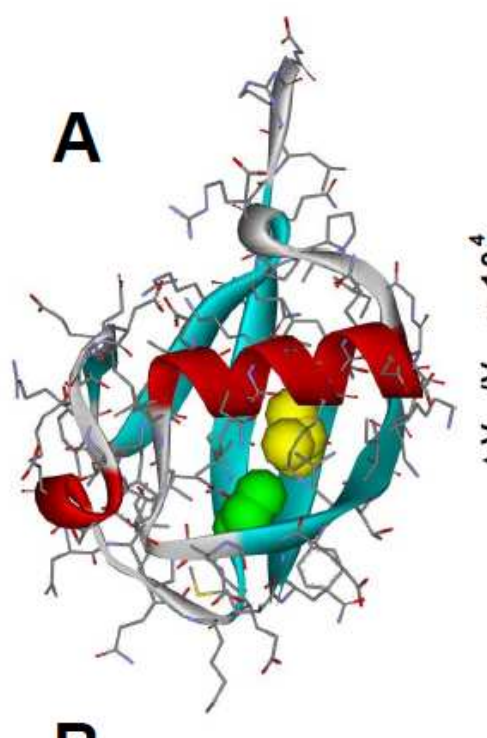

B

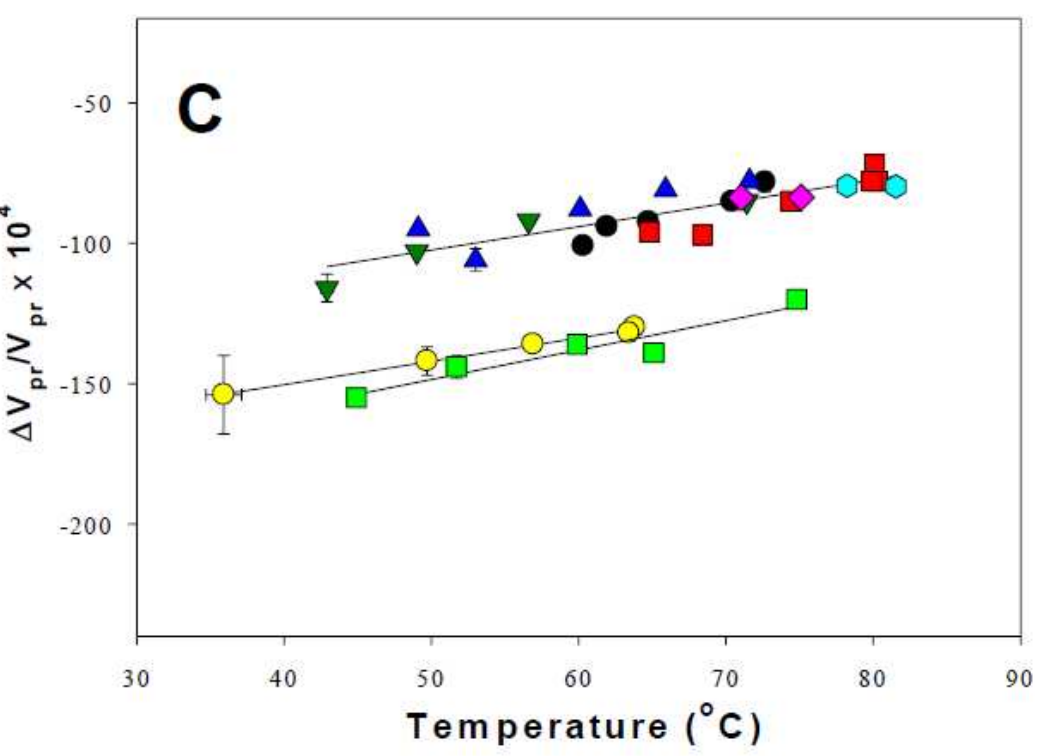

Human MQIFVKILIGKTITLEVEPSDIIENVKAKIQDKEGIPPDQQRLIFAGKQLEDGRTLSDYNIQKESTLHLVLRLRGG 6HWI * MHHHHHMQIFVKTLIGATITLEVESSDIIDNVKSKIQDKE GIP PDQQRLIFAGRQLEDGRTLSDYNIQRESTLHLVLRLRGG WI * MQIFVKILIGATITLEVESSDIIDNVKSKIQDKE GIPPDQQRLIFAGRQLEDGRTLSDYNIQRESTLHLVLRLRGG WI $\#$ MOIFVRTLTGATITLEVESSDTIDNVKSKTODKEGIPPDOOELIFAGROLEDGRTLSDYNTORESTLHLVLRLRG GA1 MQIFVKTLIGATITLEVESSDIIDKVKSKIQDKE GIP PDKQRLIFAGRELEDGRILSDYNIQRESTLQLVLELRGG GA3 MQIFVKTLIGATITLEVESSDIIDKVKSKIQDKEGIPPDKQRLIFAGRELEDGRTLSDYNIQERSTLELVLELRGG 
Figure 2

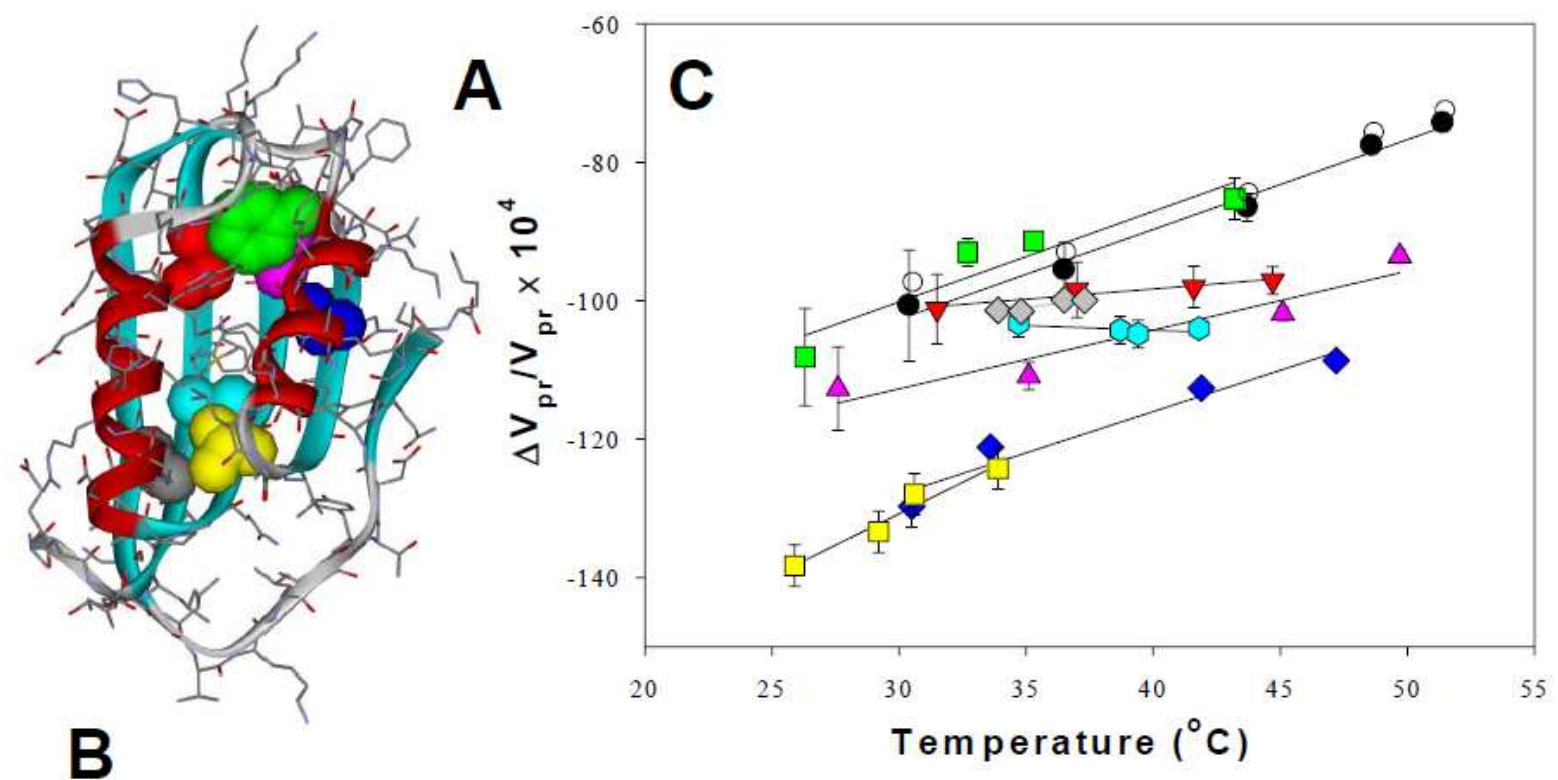

WT AEGNTLISVDYEIFGKVQGVFFRKHTQAEGKKLGLVGWVONTDRGTVQGQLQGPISKVRHMQEWLETRGSPKSHIDKANFNNEKVILKLDYSDFQIVK GA2 AEGNTLISVDYEIFGKVQGVFFRKHTQAEGKKLGLVGWVONTDRGTVQGKLQGPISKVREMOKWLETRGSPESHIDKANFKNEKVILKLDYSDFQIVK 
Figure 3

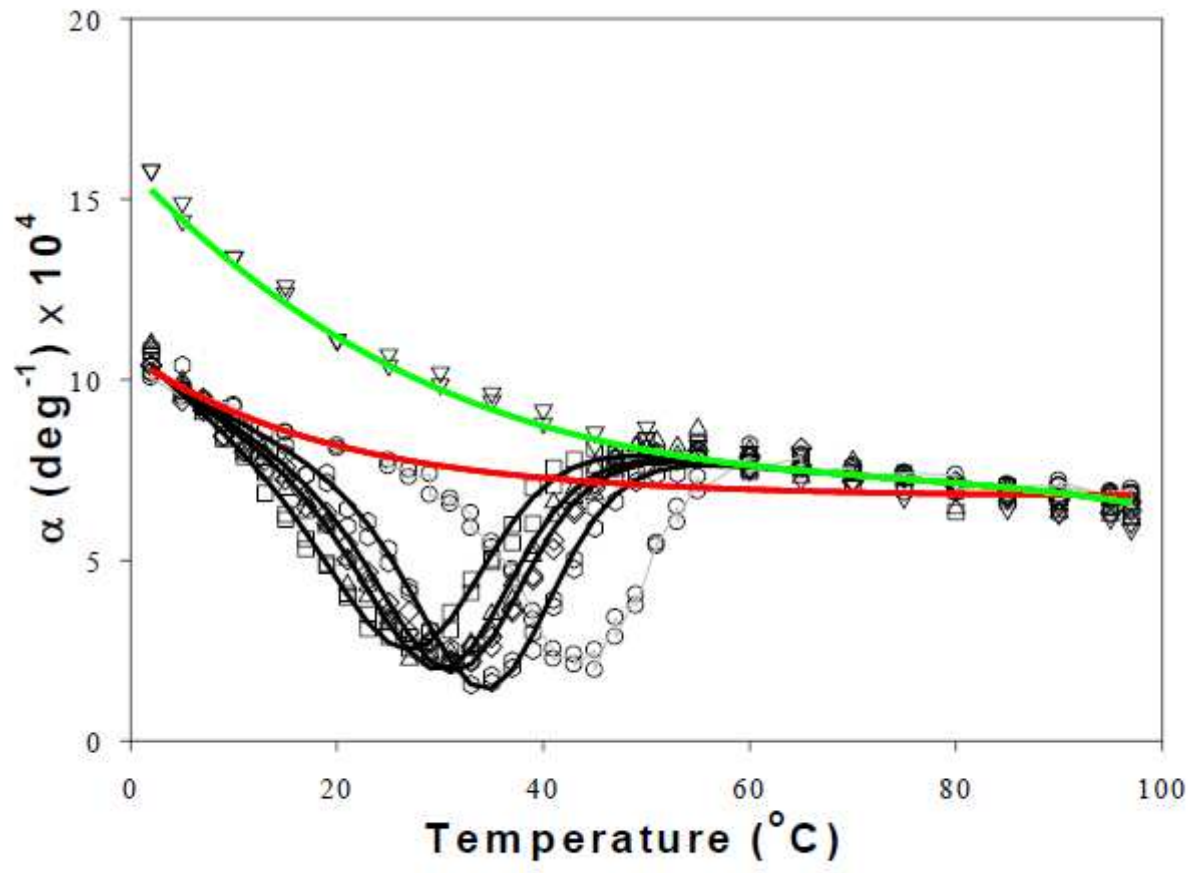


Figure 4

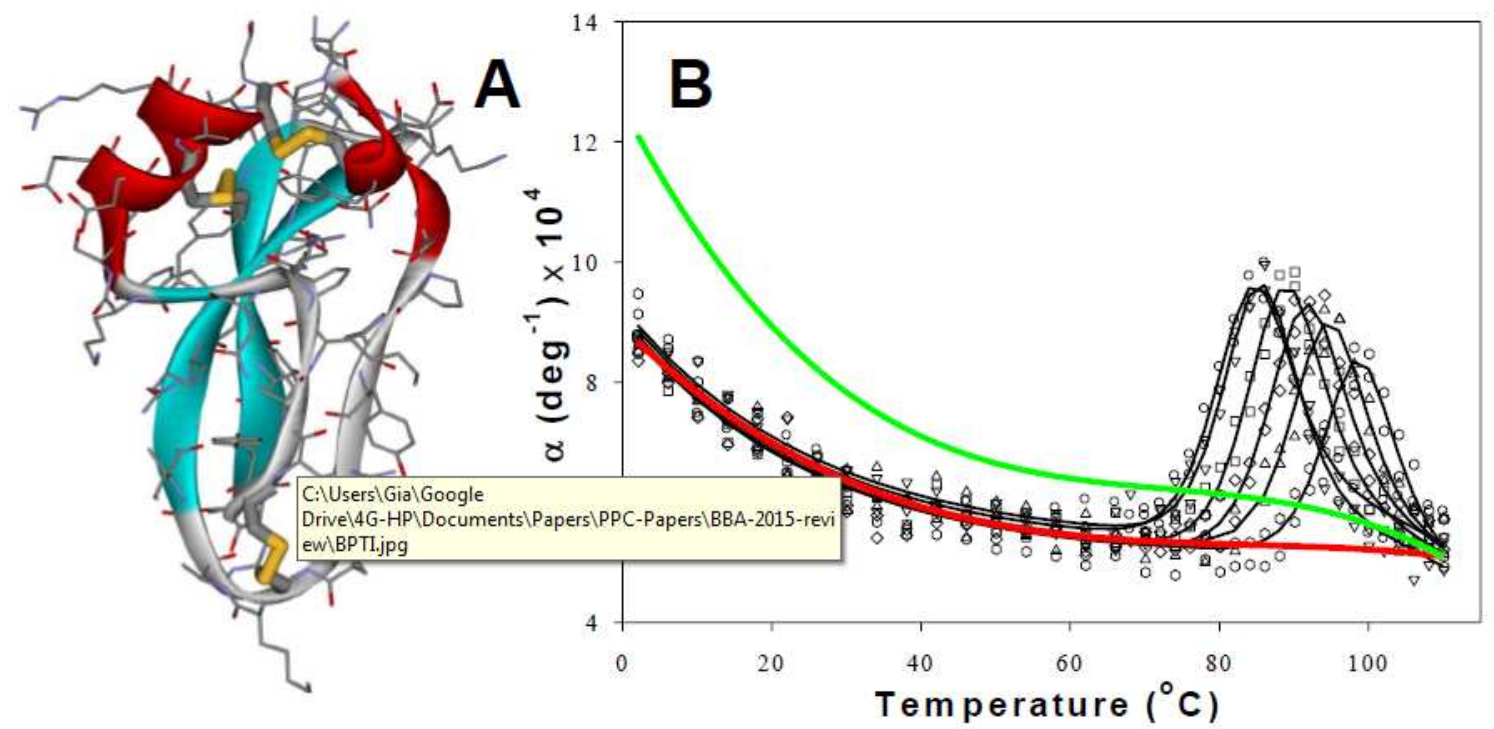


Figure 5
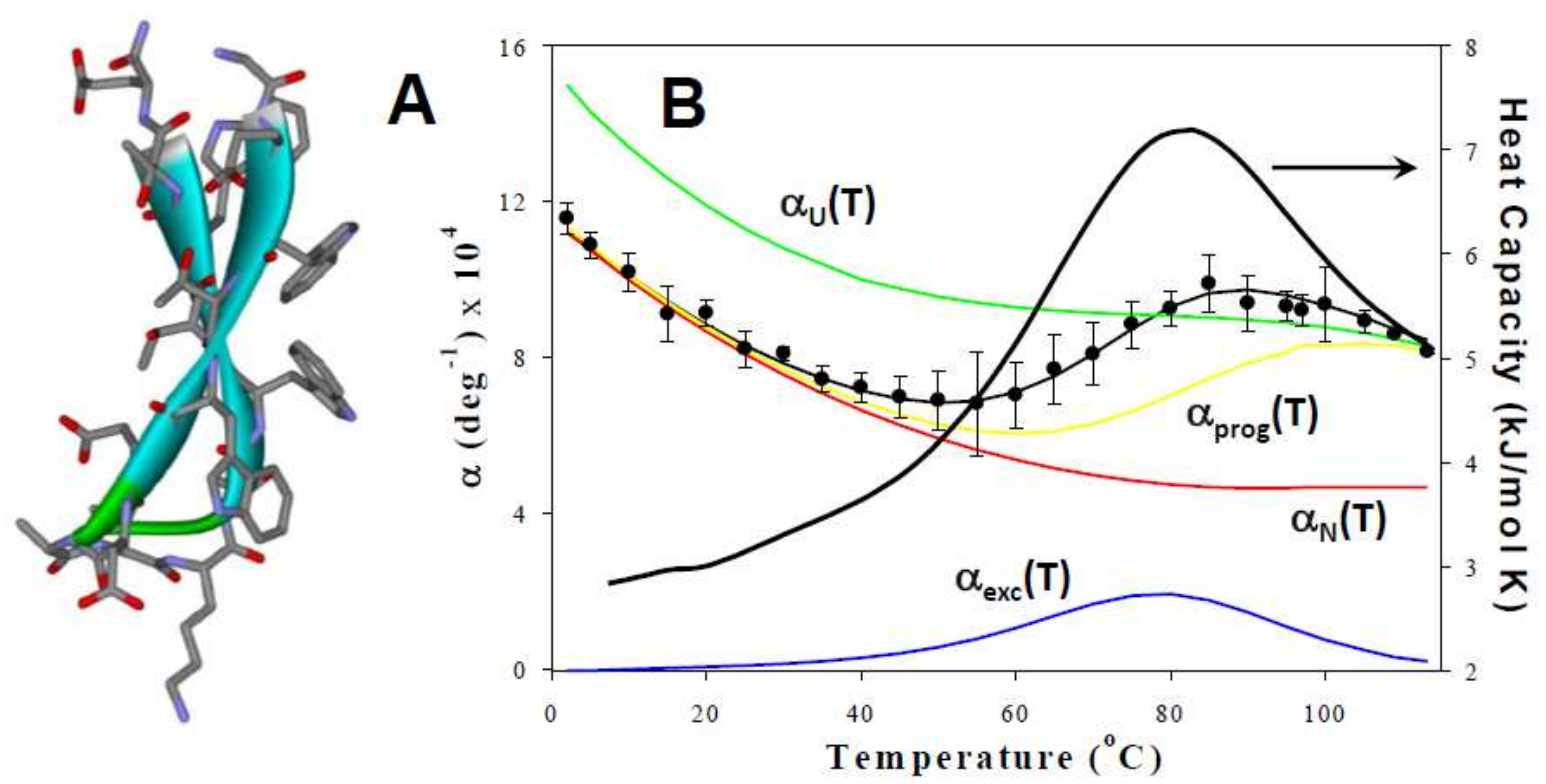